\title{
Awareness Promotion about Problem Gambling for Casino Employees: An Asian Experience
}

Irene Lai Kuen Wong*, Mina Poon

Department of Applied Social Sciences, The Hong Kong Polytechnic University, Hong Kong

*Corresponding author; email: ssilkw@inet.polyu.edu.hk

\begin{abstract}
This paper evaluates the impact of an awareness program for Asian casino employees. Contents of the program included the following topics: (1) Understanding the nature of casino games, and the notion of 'randomness' in gambling activities, (2) Examining the relationship between 'chance' and excessive gambling, (3) Defining 'safe' gambling, (4) Identifying the signs of problem gambling, (5) Deciding when and how should a casino employee offer help to problem gamblers in distress. Results indicated that the participants increased their knowledge about chance and randomness in gambling could better detect the manifestation of problem gambling, became more confident of when and how to offer help to excessive gamblers, and were convinced that responsible gambling practices would be beneficial to casinos and to patrons. The knowledge gained from the workshop was retained by participants at a follow-up undertaken after 12 months of the training. Participants' competence in offering information and help to gamblers in distress was also maintained. The workshop also generated the introduction of a venue-based customer care project. The implications of these results on responsible gambling policies are discussed.
\end{abstract}

Key words Casino $\cdot$ Awareness Promotion $\cdot$ Responsible Gambling

\section{Introduction}

In most Asian countries, responsible gambling strategies in casinos or in other gaming sectors are not mandatory. However, over the last few years, there has been increasing pressure for gambling operators to improve ethical gaming practices. This new perspective mainly originates from changing public expectations about the role of gambling operators in reducing the potentially negative consequences of their gambling products. The rapid 
expansion of legalized gambling, and the increase in the prevalence of pathological gambling in many Asian countries has contributed to changing public opinion in favor of the adoption of socially responsible attitudes (Wong, 2006).

Responsible gambling refers to policies and practices designed to prevent and/or reduce potential harms associated with gambling (Blaszczynski et al., 2004). These policies and practices incorporate a diverse range of interventions developed to promote patron and gaming employee protection, community and customer awareness, and access to efficacious treatment. In Asia, current responsible gambling programs (e.g. signage, player information leaflets, staff awareness training, employee assistance initiatives, customer care programs and self-exclusion options) are designed and adapted from strategic interventions developed in western jurisdictions including Canada, the United States, the United Kingdom and Australia (Wong, 2006). These initiatives can be broadly categorized into harm minimization and customer protection strategies (Hing, 2001). The former aims to reduce the potential harmful impacts associated with gambling. The latter safeguards consumer rights and enhances fair trading.

According to Hing (2001), harm minimization strategies include self-exclusion programs, signage on safe or responsible gambling, imposing limits on check cashing, no ATMs in gambling areas, staff detection of problem gambling, and referrals to treatment agencies. Consumer protection strategies include responsible advertising, and providing customer information about gambling products, such as how gambling activities operate, probabilities of winning and losing, and handling of disputes and malfunctioning of gaming machines. Responsible gambling practice also provides financial and in-kind support for initiatives that address excessive gambling. Examples are funds and donations granted to awareness and prevention programs and gambling studies, and other types of non-financial support for research on compulsive gambling.

Concerns and expectations of responsible gambling practices in different types of gambling forms and venues vary greatly across international jurisdictions. For example, while many western casinos have responsible gaming training, few bingo and lottery vendors have joined these programs (Derevensky et al., 2004). In Australia, there is more concern expressed about electronic gaming machines in registered clubs than at casinos (Blaszczynski at al., 2001).

Casinos in many Asia-Pacific localities, including New Zealand, Singapore, Macau, Taiwan and Japan, often attract much greater public concern and media attention (Wong, 2006). Debate about whether casinos and public health are compatible is ongoing in areas where casino gambling is or will be legalized. While governments may anticipate that casinos will generate substantial gambling revenues and economic benefits, the general public has concerns about the likelihood of increases in the incidence of problem gambling and other social ills. There has been growing demand for more ethical gaming practices and tighter governmental regulation. In response to heightened community expectations and to enhance the image of their casinos, some Asian casinos have developed responsible gambling initiatives without legislative intervention. For example, in recent years a few Chinese casinos and most of the Las Vegas style casinos in Macau have adopted self-regulatory measures such as signage, warnings against underage gambling, 
brief awareness training for dealers, employee assistance programs and player information about help lines. The casino operators attempt to address negative externalities of casino business in order to make it sustainable in the long term.

This paper evaluates the impact of a non-mandatory awareness promotion workshop which was designed for 83 gaming employees recruited from two Asian casinos. In keeping with many staff training programs on awareness promotion reported in the literature (e.g. Ladouceur et al., 2004; Shatley et al., 2006), the workshop content included the following topics: (1) increasing understanding of the notions of chance and randomness, (2) recognizing the manifestations of problem gambling, (3) teaching skills to cope with excessive gamblers, and (4) providing an overview of responsible gaming strategies implemented in many western casinos.

\section{Method \\ Participants}

Trainers were invited to design and provide awareness training for gaming employees working at two Asian casinos in December 2005. A total of 83 casino employees joined the 4-hour training session. They were selected by the casino training department with endorsement granted by senior executives. The trainers were not informed about the selection criteria used.

Participants were required to complete a pre-workshop and post-workshop questionnaire. Three of the initial 83 participants failed to complete the pre-training assessment. As a result, 80 questionnaires were distributed, and 77 completed questionnaires were collected. Only 63 complete and matched pre- and postworkshop questionnaires were available for data analysis due to inconsistent use of personal identification codes or unclear handwriting. The final response rate was $75.9 \%$.

The majority of these 63 participants were men (men: $69.8 \%, n=44$; women: $30.2 \%, n=19)$. Their ages ranged from 25 to 64 years. Almost half of the participants $(49.2 \%, n=31)$ were aged between 35 and 44 years, $16(25.4 \%)$ were aged between 45 and 54 years; $12(19.0 \%)$ between 25 and 34 years, and only four $(6.3 \%)$ were over 55 years. A high proportion of the participants $(87.3 \% ; n=55)$ were managers, and eight $(12.7 \%)$ were executives. The length of time working in the gaming industry varied from 8 to 34 years. On average, the participants worked 58.5 hours every week, and 46 of them $(73.0 \%)$ spent at least $50 \%$ of their time 'on the floor', having direct contact with customers.

\section{Procedure and measures}

Before the workshop training session, participants completed a self-administered questionnaire which was modified from a questionnaire designed by Ladouceur et al (2004). It assessed participants' assessing their knowledge of excessive gambling, their perceived role towards problem gamblers, and self-assessed competence in coping with gamblers in distress. For example, they were asked to rate themselves on a four-point scale which assessed their understanding of the signs of problem gambling ( 1 - I "totally disagree" that I have good understanding of the signs of problem gambling, 2 - "disagree", 3 - "agree", 4 - "totally agree"). They were also 
asked if they expected themselves to offer help to gamblers in distress $(0$ - "no", 1 - "yes"), and how frequently they had offered help to gamblers in the past year (1 - "never", 2 - "rarely", 3 - "sometimes", 4 - "very often").

A similar questionnaire was completed after the workshop. Several openended questions were also included to gather participants' views on responsible gambling policies and strategies. For example, they could freely comment on both the benefits and costs of implementing such programs, and could also indicate their readiness to support customer assistance initiatives. Participation was voluntary and anonymous.

During the training session, the participants were first split into small groups to share their views on signs of problem gambling and safe gambling. A lecture on the definition and the symptoms of excessive gambling was given, followed by a 15-minute video showing how to approach and cope with a problem gambler, and then various ways to offer help to gamblers were suggested and discussed.

In a subsequent skill training session, the participants were given an opportunity to practice a range of coping techniques via role-plays followed with debriefing. Training of techniques focused on how to identify customers with a gambling problem, how and when to approach these customers, and how to provide information on problem gambling and help. Discussion was also focused on the roles to be played by different levels of gaming floor staff, including dealers, 'pit bosses', supervisors, managers, security and surveillance staff and customer service personnel. The workshop closed with an overview of responsible gambling strategies commonly implemented in western jurisdictions, including information brochures, staff awareness training, self-exclusion options, customer care and employee assistance programs. A brief discussion on the applicability of these strategies in their casinos was also included.

\section{Results and Discussion}

The majority of the participants $(95.2 \%)$ had not attended an awareness workshop previously. They appeared to have been chosen because they had experience and competence in handling customers' queries and problems. It is a possibility that those who attended the workshop were those ready to offer help to patrons, and those not being resistant to awareness training. It is likely that the apparent open attitudes towards responsible gambling practices had positive effects on the post-training evaluation, and the subsequent development of customer assistance strategies.

In brief, the participants' feedback was very positive. A high proportion of participants $(96.8 \%)$ were satisfied with the workshop as a whole. The majority found the information provided concerning manifestations of excessive gambling, and how to cope with these individuals useful to their job as a casino employee (71.4\% and $66.7 \%$ of respondents, respectively). They liked exchanging ideas $(65.9 \%)$, and found group discussion and role-plays stimulating and interesting (71.4\%). A high percentage $(69.8 \%)$ of the participants remarked that it would be necessary to include awareness training in the casino employees' orientation programs.

At the end of the workshop, $61.7 \%$ of participants described themselves as being more empathetic, and feeling less helpless about coping with gamblers in 
distress $(58.7 \%)$. They realized that they had a role to play in providing information in regard to various sources for help for excessive gamblers $(60.0 \%)$. They were eager to discuss how to further develop a systematic venue-based customer care program.

In order to assess if there were significant changes in the participants' knowledge of excessive gambling and when and how to intervene with customers showing evident signs of problem gambling, paired $t$-tests (without corrections made) on the pre- and post-training data were computed. Results indicate a significant increase in participants' understanding of the signs of problem gambling $(t=-2.21, p<0.01)$, and the notions of chance and randomness in casino games $(t=$ $-2.55, p<0.01)$. Interestingly, they became more convinced that gamblers would not be able to win more money even if they tried to use a 'system' or strategy when they gambled $(t=-4.35, p<0.001)$. Upon completion of the workshop, the participants were more willing and competent to suggest help to gamblers who were in distress $(t=1.60, p<0.05)$. They reported feeling more confident in choosing the most suitable moment to intervene $(t=-1.64, p<0.05)$.

The majority of participants $(80.9 \%)$ left the workshop with a better understanding of customer assistance initiatives $(t=3.2, p<0.01)$, and responsible gambling practices in general. They agreed that all gaming floor staff should attend an awareness promotion program (79.4\%). Nearly three quarters $(74.6 \%)$ of participants were convinced that with the development of responsible gambling strategies, such as customer care and protection programs, the reputation of the casinos would improve in the longer term. They could envisage the benefits of implementing ethical gaming practices in the gaming operations, and were prepared to play an active role in such implementation.

Among the participants $(n=20,31.7 \%)$ who responded to a twelve-month follow-up exercise, changes in knowledge were maintained, including knowledge about the notion of chance and randomness in gambling (98.4\%) and symptoms of excessive gambling (96.8\%). They reported that they felt competent in offering information to an excessive gambler in distress (95.2\%), and 68\% reported that they did approach and offer help to gamblers more often than before.

Within a period of twelve months after the completion of the workshop, awareness training had been integrated as an essential component of the orientation programs for the newly recruited gaming floor staff. A customer care project was developed with properly trained staff to answer enquiries on gambling problems from patrons and their family members via the casino help lines. Gamblers in distress were also referred to professional counseling and other services. Other responsible gambling strategies were also implemented including use of information pamphlets and posters, self-exclusion options, and warnings about minimum age restriction for casino entry and playing. All these responsible gaming measures were designed and implemented under the trainers' guidance and advice.

During the follow up visits to the casinos in 2006 and 2007, the trainers were offered an opportunity to attend several orientation sessions organized for the newly recruited gaming floor staff $(n=389)$, and observed firsthand how the workshop participants integrated awareness training in these orientation programs. They started with giving a brief talk on the signs of problem gambling. Then the 
new comers were taught how to identify excessive gamblers, and how and when to report a case to a supervisor. In addition to their managerial responsibilities, a dozen of the participants had taken up the roles of awareness trainers in these orientation programs.

In a consultation session, the leaders of the customer care project made a report on the number of information leaflets distributed to customers, and the number of calls received from gamblers and their family members via the help lines. They also described enquiries about the self-exclusion program, and the number of temporary bans from casino gambling imposed on members who had left their children unattended while they were playing inside the casinos. They actively sought our comments and advice for further improvements. Almost half $(48 \%)$ of the workshop participants and the senior management remarked that responsible gambling policies and programs should be made mandatory. They believed that these ethical practices would bring benefits not only to the customers, the casino employees, but would also help making casino gambling industry a sustainable business.

To our knowledge, this was the first industry-initiated awareness promotion program based on responsible gambling conducted in Asia. The findings give support to the benefits of implementing such initiatives in other Asian casinos and other gaming sectors. At the beginning of the workshop, about a quarter $(23 \%)$ of participants felt concerned about their customers' excessive gambling and other gambling-related difficulties. They felt helpless towards gamblers in distress. After completion of the workshop, the majority of participants gained a new vision of ethical gaming practices, and reported feeling more competent and less frustrated in coping with problem gamblers. They were ready to apply the techniques of how to detect, to approach and offer help to an excessive gambler in their workplace. Above all, the changes were maintained at the follow-up phase, and were transformed into actions. Furthermore, under the guidance of the trainers, a dozen of the participants began to provide brief awareness training to newly employed gaming floor staff.

The participants expressed a need for a refresher course in the future, and the most senior executives sought advice on how to further improve the existing responsible gambling strategies. All these changes indicate that gaming employees have shown increasing recognition of the need to implement responsible gambling programs at their casinos. On the basis of these findings, there is optimism that more casinos in Asia will develop and implement similar awareness training programs and responsible gambling measures in the near future to meet changing public and governmental expectations.

To conclude, this awareness training generated very positive outcomes. The casino operators and the participants were open-minded and enthusiastic about developing responsible gambling measures at their casinos. These factors contributed enormously to the positive outcomes of the training workshop and the development of subsequent customer care strategies. Unfortunately, only one-third of the employees participated in the follow-up exercise because of staff turn over. Other limitations of this efficacy evaluation were the absence of a control group and helping skill tests in the real casino environment. Future efficacy investigation should consider addressing these limitations. 


\section{Acknowledgments}

The authors would like to thank participating casino employees for their involvement in this study.

\section{References}

Blaszczynski, A., Ladouceur, R., \& Shaffer, H. (2004). A science-based framework for responsible gambling: The Reno model. Journal of Gambling Studies, 20, 301-317.

Blaszczynski, A., Sharpe, L., \& Walker, M. (2001). The assessment of the impact of the reconfiguration of electronic gambling machines as harm minimization strategies for problem gambling. University of Sydney: University of Sydney Gambling Research Unit.

Derevensky, J. L., Gupta, R., Messerlian, C., \& Gillespie, M. (2004). Youth gambling problems: A need for responsible social policy. In J. L. Derevensky \& R. Gupta (Eds.), Gambling problems in youth: Theoretical and applied perspectives (pp. 245). New York: Kluwer Academic.

Hing, N. (2001). Changing the odds: A study of corporate social principles and practices in addressing problem gambling. Journal of Business Ethics, 33, 115-144.

Ladouceur, R., Boutin, C., Doucet, C., Dumont, M., Provencher, M., Giroux, I. (2004). Awareness promotion about excessive gambling among video lottery retailers. Journal of Gambling Studies, 20, 181-185.

Shatley, J., Hestermann, D., Ladouceur, R., Whelan, J., \& Braunlich, C. (2006). Harrah's national responsible gaming programs: Policy, technology, training, and evaluation. In Conference proceedings - An international conference on gaming industry and public welfare 2006 (pp. 137144). China Center for Lottery Studies, Peking University, Macao Polytechnic Institute and Institute for Tourism Studies.

Wong, I. L. K. (2006). Challenges confronting Asian gaming industry: developing responsible gaming training and related initiatives. In Conference proceedings - An international conference on gaming industry and public welfare 2006 (pp. 179-188). China Center for Lottery Studies, Peking University, Macao Polytechnic Institute and Institute for Tourism Studies. 